\title{
Porokeratosis - report of two cases
}

\author{
Andreea Anton ${ }^{1}$, Laura Stătescu ${ }^{1,2}$, Anca Zbranca ${ }^{1,2}$, Dan Vâță ${ }^{1,2}$, Tatiana Țăranu ${ }^{2,3}$, \\ Adriana Pătraşcu ${ }^{1, *}$, Laura Gheucă Solovăstru ${ }^{1,2}$
}

${ }^{1}$ Dermatology Department "Sf. Spiridon" Emergency Clinical Hospital lași, România, ${ }^{2 "}$ Grigore T. Popa" University of Medicine and Pharmacy lași, Romania, ${ }^{3}$ Department of Dermatology "CF" Clinical Hospital lași, Romania

\begin{abstract}
The term of porokeratosis refers to a group of specific disorders of keratinization clinically characterized by atrophic patches surrounded by an elevated hyperkeratotic border and histologically by the cornoid lamella. Multiple clinical variants of porokeratosis are recognized. Herein we report two cases of porokeratosis, one case of disseminated superficial actinic porokeratosis and the other one of atypical porokeratosis of Mibelli. Mibelli typically has an early onset and the lesions are large and isolated. In both cases, the diagnosis was confirmed by dermatoscopy and skin biopsy. The management of porokeratosis includes many options. In both cases, sun protection, emollients and keratolytics were prescribed with significant improvement.
\end{abstract}

Keywords: porokeratosis, disseminated superficial actinic porokeratosis, classic porokeratosis of Mibelli, atypical Mibelli form, cornoid lamella

\section{Introduction}

Porokeratosis represents a rare, chronic, progressive skin disorder, most often seen in males, clinically characterized by atrophic patches surrounded by a hyperkeratotic ridgelike border. The histological marker is represented by the cornoid lamella [1].

Five clinical variants are recognized: disseminated superficial actinic porokeratosis and its non actinic variant, classic porokeratosis of Mibelli, linear porokeratosis, porokeratosis palmaris et plantaris disseminata and punctate porokeratosis [2].

The etiology and pathogenesis remain unclear but, studies have identified several risk factors for the development of porokeratosis including genetic inheritance (an autosomal

Received: October 2016; Accepted after review: November 2016; Published: December 2016.

*Corresponding author: Adriana Pătraşcu, MD, Dermatology Department "Sf. Spiridon" Emergency Clinical Hospital, 1 Independentei Blvd., Iași, România

Email: patrascuai@yahoo.com dominant inheritance pattern with incomplete penetrance), immunosuppression, ultraviolet radiation and trauma [3, 4].

Disseminated superficial actinic porokeratosis is characterized by small (0.5 -1 $\mathrm{cm})$, annular, brown macules, with raised peripheral ridges, symmetrical, usually asymptomatic or slightly itchy [5]. Porokeratosis Mibelli initially appears with small, asymptomatic, brown, hyperkeratotic lesions that will slowly expand over a period of time, located on extremities [6].

\section{Case 1}

A 55 year-old woman, retired farmer, presented with multiple, slightly itchy, lesions on her forearms and legs evolving for two years. The patient had no family history of porokeratosis. She was diagnosed with recurrent depressive disorder and CEAP 3 venous insufficiency. On physical examination, the patient presented multiple, annular, brown, 
atrophic, hyperkeratotic, $0.5-1 \mathrm{~cm}$ in diameter, minimally scaly papules (Figures 1 and 2).

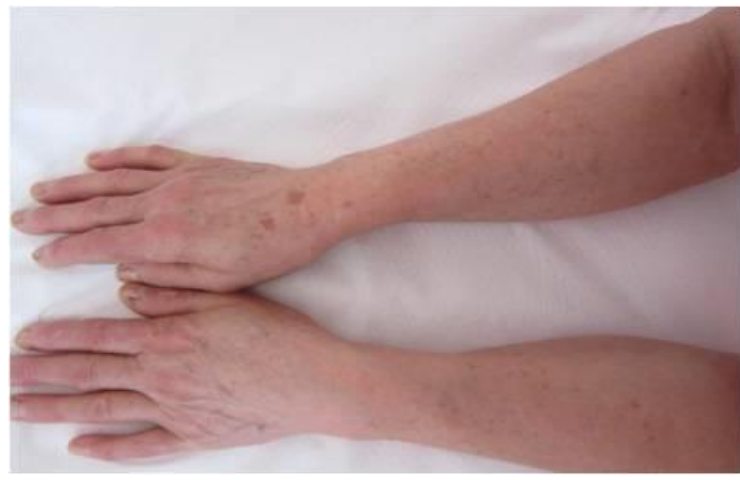

Fig. 1. Clinical appearance: annular, brown, hyperkeratotic $0.5-1 \mathrm{~cm}$ in diameter papules on the forearms.

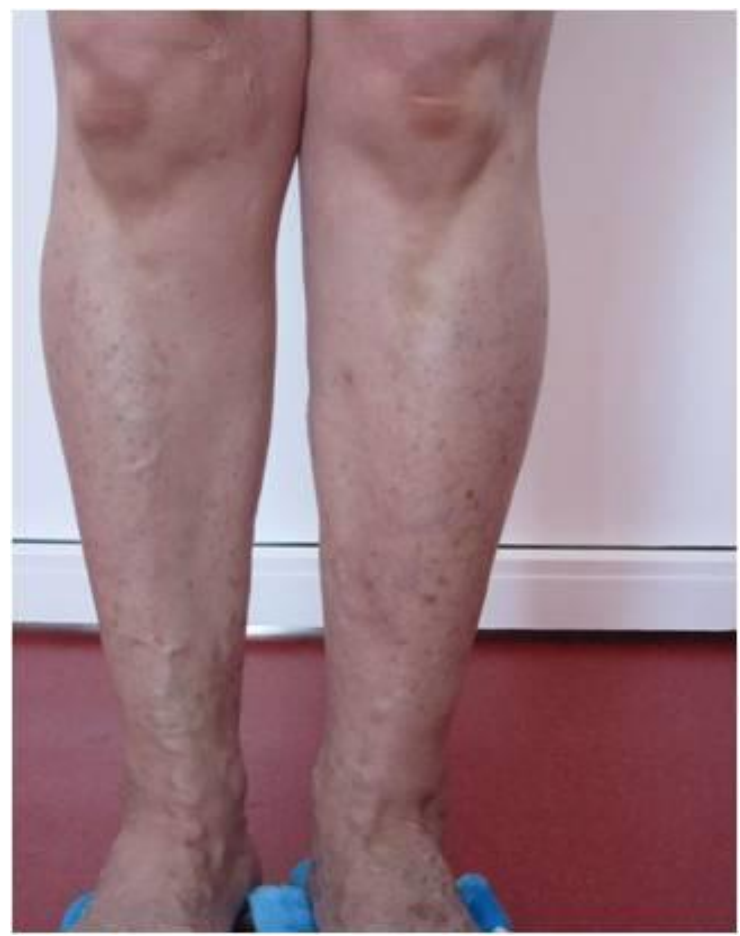

Fig. 2. Clinical appearance: annular, brown, hyperkeratotic $0.5-1 \mathrm{~cm}$ in diameter papules on the legs.

Dermatoscopy showed a "white track" at the periphery of the lesion, corresponding to the cornoid lamella; the center showed a white homogeneous area, corresponding to the atrophic epithelium and red dots corresponding to enlarged capillary vessels (Figure 3).

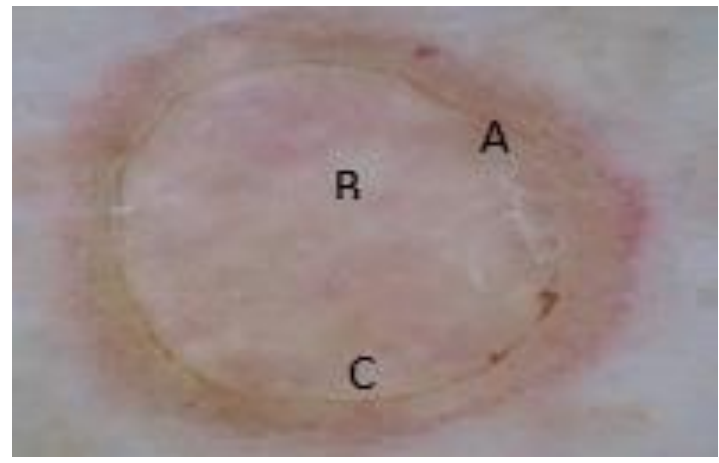

Fig. 3. Dermatoscopy: peripheral cornoid lamella (A), atrophic epithelium (B), enlarged capillary vessels $(C)$.

Histopathology of skin revealed a hyperkeratotic epidermis with columns of parakeratotic stratum corneum cells (cornoid lamella) extending at an angle away from the center of the lesion; no granular layer is seen within the parakeratotic column; the papillary dermis contains a mild lymphocytic infiltrate and dilated capillaries (Figure 4).

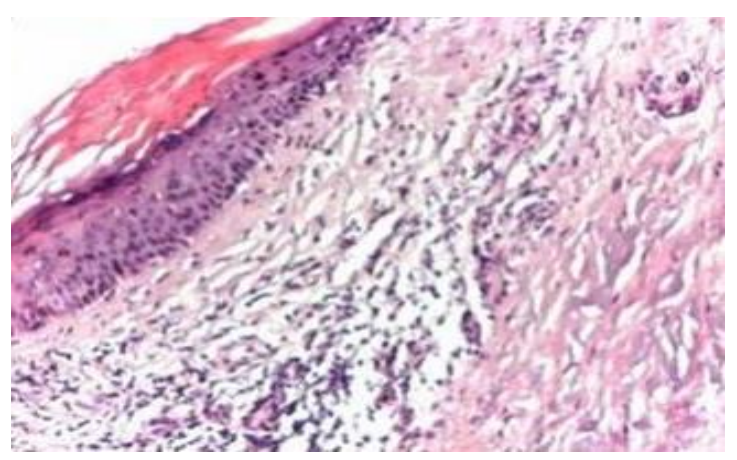

Fig. 4. Histopathology exam: cornoid lamella, absence of the granular layer, lymphocytic infiltrate in the papillary dermis (HE, x100).

On the basis clinical examination, dermatoscopy and histopathological examination, a diagnosis of disseminated superficial actinic porokeratosis was made. Protection from the sun, use the emollients with $5 \%$ urea and keratolytics (20\% urea), were prescribed for the management of the lesions, with significant improvement of the lesions within one month.

\section{Case 2}

A 76 year-old man presented with a 1 year history of progressive, slightly pruritic lesions on both legs. The patient had no significant 
family history and was diagnosed with hypertension and asthma. The patient had no history of prolonged sun exposure. Physical examination revealed multiple, erythematouskeratotic papules, with slightly elevated edges, covered with white, adherent scales, measuring 1-2 cm, located on both legs (Figures 5 and 6).

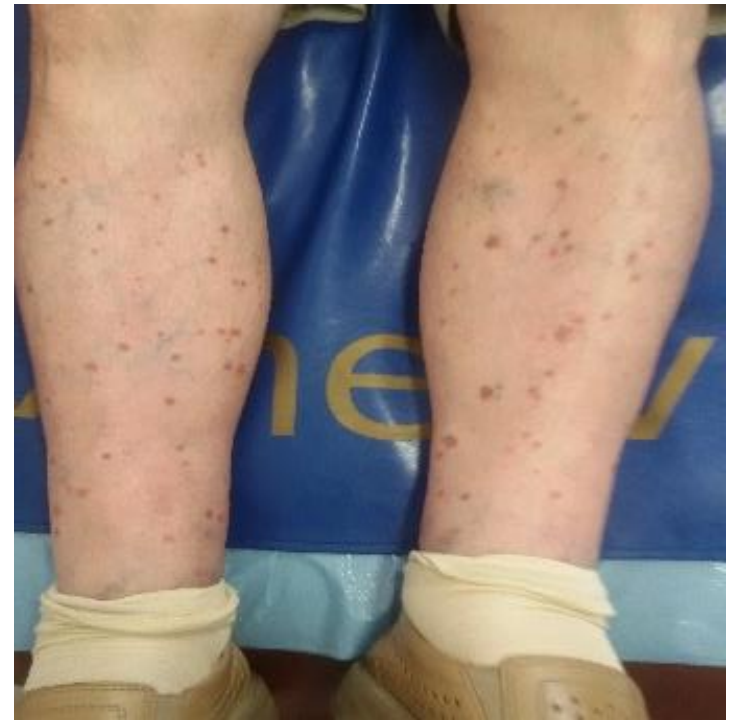

Fig. 5. Clinical appearance: erythematous-keratotic, scaly papules on both legs.

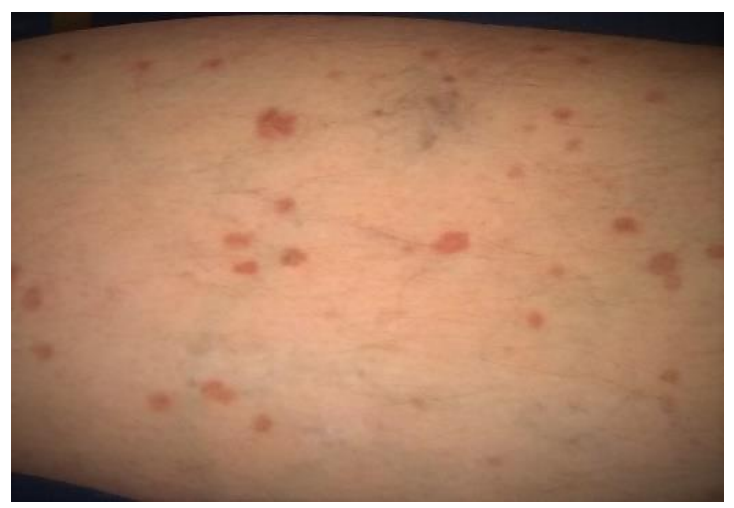

Fig. 6. Clinical appearance, close-up of lesions: slightly elevated edges and atrophic center.

Dermatoscopy revealed a white track structure surrounding the entire perimeter of the affected area - corresponding to the cornoid lamella. The center showed a brownish pigmentation and red dots, lines and globules corresponding to vessels (Figure 7).

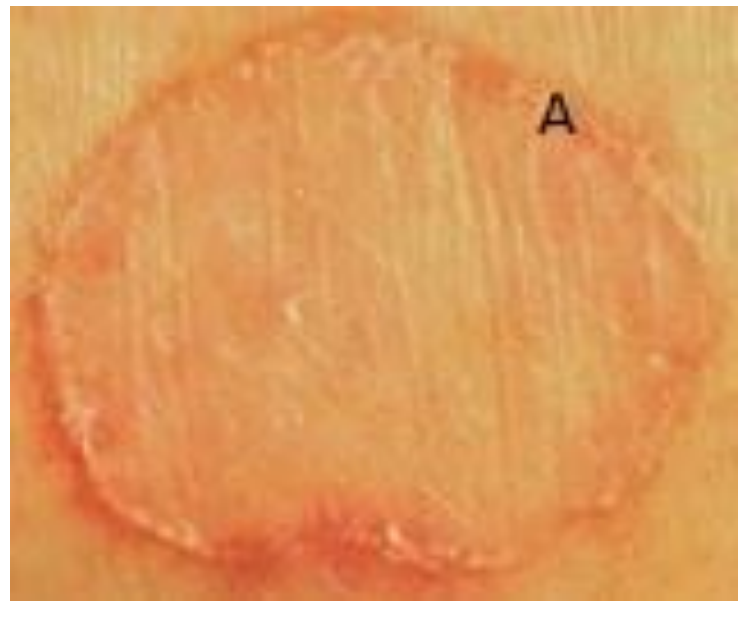

Fig. 7. Dermatoscopy: peripheral cornoid lamella (A).

Histopathological examination revealed the cornoid lamella with invagination of the epidermis; interruption of the granular layer; dense lymphocytic infiltrate, with no atypical cells, in the superficial dermis beneath the cornoid lamella (Figure 8).

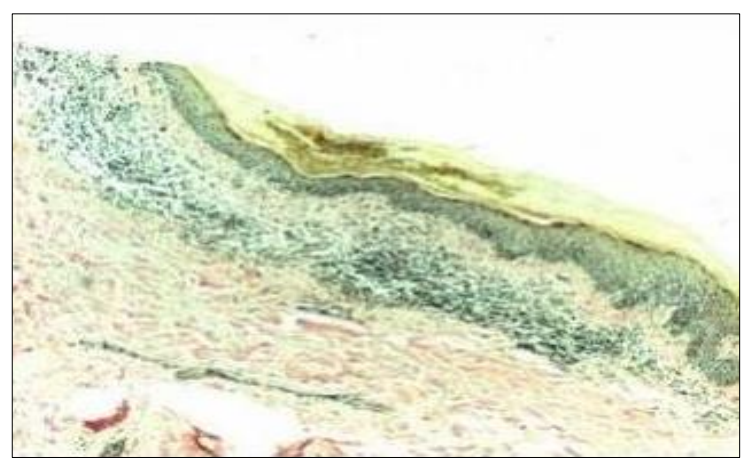

Fig. 8. Pathological exam: cornoid lamella with underlying interrupted granular layer; dense lymphocytic infiltrate in the superficial dermis (van Gieson stain, x40).

The clinical and paraclinical examinations established the diagnosis of porokeratosis of Mibelli. The treatment prescribed, considering the age of the patient and the availability of the medication, was sun protection and topical keratolytic $(20 \%$ urea) which provided improvement of the lesions within three weeks. The patient was satisfied with this result and refused to pursue more therapy, after we explained the other therapeutic options, the advantages and the disadvantages. 


\section{Discussion}

The most common variants of porokeratosis are the disseminated superficial actinic porokeratosis and the classic porokeratosis of Mibelli.

The differential diagnosis of psoriasis, porokeratosis and cutaneous T-cell lymphoma mimicking porokeratosis was considered $[7,8]$.

The lesions are most often present at patients with suggestive family history and chronic sun exposure and the typical debut is in the third-fourth decade of life in disseminate superficial actinic variant and in childhood or young adulthood in porokeratosis of Mibelli. In both cases, there was no suggestive family history and the age of the second patient was atypical. Also, he had no history of prolonged sun exposure and the lesions were not classic porokeratosis of Mibelli but the histopathological analysis confirmed the diagnosis [9].

The particularity of the first case consists in association of recurrent depressive disorder, which is a contraindication to oral retinoids and

\section{References}

1. Wolff K, Johnson R, Saavedra A. Fitzpatrick's Dermatology in General Medicine. Volume 1. $7^{\text {th }}$ Edition. The McGraw-Hill Companies Inc., 2008.

2. Pătraşcu V. Boli dermatologice si infectii sexual-transmisibile. $3^{\text {rd }}$ Edition. Craiova: Sitech, 2014.

3. Kanitakis J, Euvrard S, Faure M, Claudy A. Porokeratosis and immunosuppression. Eur $J$ Dermatol. 1998; 8(7):459-466.

4. Sertznig $P$, von Felbert V, Megahed $M$. Porokeratosis: present concepts. J Eur Acad Dermatol Venereol. 2012; 26(4):404-412.

5. Murase J, Gilliam AC. Disseminated superficial actinic porokeratosis co-existing with linear and verrucous porokeratosis in an elderly woman: Update on the genetics and clinical expression of porokeratosis. J Am Acad Dermatol 2010; 63(5):886-891.

6. Koley S, Sarkar J, Choudhary S, Dhara S, Choudhury M, Bhattacharya S. Different morphological variants of hypertrophic porokeratosis and disseminated lesions of porokeratosis of Mibelli: a rare co-existence. topical retinoids on large areas administration [10].

The management of porokeratosis includes many options but it must be individualized, based on the characteristics of the lesions. Methods used include the following: emollients, keratolytics, topical 5fluorouracil, topical vitamin D3 analogues, topical retinoids, oral retinoids, cryotherapy, surgical methods and laser treatment [11].

The prognosis is generally good but the lesions should be carefully monitored for the development of cutaneous malignancies as malignant degeneration has been reported in almost all forms of porokeratosis [12].

\section{Conclusion}

Porokeratosis represents a condition frequently overlooked and it should be recognized and diagnosed correctly. The variety of therapeutic options reveals that there is no perfect treatment, both safe and effective.

Indian J Dermatol Venereol Leprol 2011; 77(2):199-202.

7. Wallner JS, Fitzpatrick JE, Brice SL. Verrucous porokeratosis of Mibelli on the buttocks mimicking psoriasis. Cutis 2003; 72:391-393.

8. Brenemann DL, Brenemann JC. Cutaneous Tcell lymphoma mimicking porokeratosis of Mibelli. J Am Acad Dermatol 1993; 29:10461458.

9. Kempf W, Hantschke M, Kutzner H, Burgdorf WHC. Dermatopathology. Springer, 2007.

10. Hayes J, Koo J. Depression and Acitretin: A True Association or a Class Labeling. JDD 2011; 10(4):409-412.

11. Lebwohl MG, Heymann WR, Berth-Jones J, Coulson I. Treatment of Skin Diseasecomprehensive therapeutic strategies. $4^{\text {th }}$ Edition. Elsevier Saunders, 2014.

12. Requena L, Requena $C$, Cockerell CJ. Benign Epidermal Tumors and Proliferations. In: Bolognia J, Jorizzo J, Schaffe J (Ed.) Dermatology. Volume 2. $3^{\text {rd }}$ Edition. Philadelphia: Elsevier Saunders; 2012:18011803. 